\title{
A Broad-Spectrum Computational Approach for Market Efficiency
}

\author{
Olivier BRANDOUY ${ }^{1}$ and Philippe MATHIEU ${ }^{2}$ \\ 1 LEM, UMR CNRS-USTL 8179 \\ olivier.brandouyduniv-lillel.fr \\ ${ }^{2}$ LIFL, UMR CNRS-USTL 8022 \\ philippe.mathieudifl.fr
}

The Efficient Market Hypothesis (EMH) is one of the most investigated question in Finance. Nevertheless, it is still a scientific puzzle, despite the enormous amount of research it has provoked: as instance, many recent results have shadowed the wellestablished belief that market cannot be outperformed on the long run (see as instance Detry and Gregoire (2001)); an other reason is that persistent market anomalies cannot be easily explained in this theoretical framework (Shiller 2003). One can also consider that some talented hedge-fund managers (like Jim Simons) seem to earn excess risk-adjusted rates of returns since years. Nevertheless, this last point does not reach any consensus today (Malkiel 2003).

Many formulations of the EMH have been proposed since Samuelson founding work (Samuelson 1965). We concentrate in this paper on the weak form of efficiency (Fama 1970) : "past informations are useless to predict future price changes". We therefore focus on the profitability of simple technical trading rules following in that Jensen and Benington (1969) or more recently Brock, Lakonishock, and LeBaron (1992). An extensive survey for this field is proposed in Park and Irwin (2004).

Nevertheless, we depart from previous works in many points : we first have a large population of technical, virtual agents (more than 2.6 millions) exploiting real-world data to manage a financial portfolio as chartists or technical traders would do. Very few researches have used such an enormous amount of calculus to check the EMH. Our experimental design allow for agents selection based on past absolute performance, as well as stability in performance. Of course, we take into account the datasnooping risk, which is an unavoidable problem in such broad-spectrum works, using a rigorous bootstrap Reality Check procedure (White 2000).

Whereas market inefficiencies, when including transaction costs, cannot clearly be successfully exploited, our experiments present troubling outcomes like persistent (but not statistically significant at commonly admitted levels) over performance inviting to consider again more closely the weak-form EMH.

This short version is organized as follows: section 1 presents our multi-agent system (MAS) and experimental design, section 2 briefly sum-up our results. 


\section{Methodology}

In this section we give the main features of our experimental design including the global MAS architecture, descriptions of the agents, and the statistical procedure aimed to detect potential market inefficiencies.

\subsection{MAS architecture}

In this experiment, agents represent virtual investors trading one financial commodity called a tracker". As it is generally admitted (Wooldridge 2002), the agents' fundamental characteristics in this study are an idiosyncratic decision-making process, autonomy, and reactivity to context changes. Our MAS is based on a three-stage architecture; at each stage, one can consider a kind of agents with specific aims or logic:

First stage: Strategic Agents are micro-agents playing always the same basic strategy through the entire simulation. Those basic strategies are known in the financial community as "technical trading rules". As instance, a Strategic-agent :"5-days moving average" cannot process any other operation and decide to trade or not her tracker on the basis given by her single rule.

Second stage: Family Agents are general agents defining all the formal characteristics used in the instantiation of each Strategic Agent. Each Family Agent has also to perform a ranking between each of his "children" at each time step. The Family Agent has thus the capacity to select the most successful individuals in the Strategic Agents population.

Third Stage: Meta Agents are able to mimic the behavior of various Strategic Agents according to the circumstances and the ranking given by the relevant Family Agents. As instance, a Meta Agent based on the 2-uple \{Momentum, Triangle\} will choose and mimic various instances of those Family Agents, considering some signals. We do not develop this point in this short version nor report results concerning this category of agents.

To get it clearer, let's consider one Family Agent : "Periodical Trader". This agent buys and sells the trackers at fixed periods. She is similar to speculators buying on Mondays and selling on Fridays. This agent has at least two parameters coding the dates at which she will buy and sell her trackers. If she decides to generate all possible Strategic Agents using all possible delays between 1 and 100, 10.000 "children" will be processed.

\subsection{Agent's design}

As it has been presented previously, we have implemented a large population of heterogeneous agents (2.621.932 agents, see Table 1). Such massive experiment has never been performed before, to our point of view.

Allowed operations and behavior: Each agent is allowed to trade $n$ tracker $(n \in$ $R^{+}$), that is, one financial commodity replicating exactly one market index (like 
Table 1. Agents Population

\begin{tabular}{c|r} 
Family Agent & Num. Strategic Agents \\
\hline Periodical Trader $\{n, p\}$ & 2.500 .000 \\
Indicator Trader $\{n, p\}$ & 13.928 \\
Rectangle Trader $\{n, m, p, s\}$ & 57.566 \\
Triangle Trader $\{n, m, p, s\}$ & 4.303 \\
Variation Trader $\{n, m, p\}$ & 19.960 \\
Momentum Trader $\{n, p\}$ & 1.485 \\
Moving Average Trader $\{n, p\}$ & 1.987 \\
Weighted Moving Average Trader $\{n, p, s\}$ & 1.990 \\
RSI Trader $\{n, p\}$ & 20.712 \\
Buy \& Hold Trader & 1 \\
\hline
\end{tabular}

CAC40, Dow-Jones or Nikkei). If she has not decided to hold such commodities, the agent holds cash. Therefore, each agent is in one of these situations:

- she possesses a number of trackers $>0$; in this situation we say the "Agent is in the market". Her wealth fluctuates along the market.

- she does not have any tracker or fraction of tracker, all her wealth being converted in cash; the "Agent is out-of the market" and her wealth is stable. No risk-free asset paying a low interest rate is available.

At each time step, agents receive a new information and have to decide if it is worth staying in the market or getting out : they follow systematically the signals given by their technical rules. As instance, a Strategic Agent designed as a "moving average 5, 5 " analyzes at each iteration if the 5 days moving average of past prices has crossed the price process in the top-down direction, which correspond to a "sell-signal" (resp. bottom-up and "buy-signal"). In this situation, if the gap between the 5-days moving average and the price is greater than 5\%, she will "sell" (resp. "buy"). If the gap is under 5\%, she will stay unchanged considering her portfolio. Each Strategic Agent possesses the same kind of behavior with various chartist or technical rules. Nevertheless, one has to notice than one singular agent follows systematically a "Buy \& Hold" strategy, that is, she get in the market at time $=0$ (she buys one tracker) and let her situation unchanged until the end of the simulation. This agent is our "benchmark" agent in terms of risk and return and stands for a "passive investor". Theoretically, no one can outperform this agent on the long run assuming the EMH holds.

Each agent is endowed with the same amount of cash at the beginning of the simulations. If an agent looses all her endowment during an experiment, since borrowing is avoided, she is withdrawn from the market.

Trading is subjected to transaction costs at a $0.5 \%$ level.

The simulations are based on real daily data from the Euronext Paris Stock Exchange between 1988 and 2005. The traded tracker perfectly replicates the CAC40 index. Agents have only access to past values of this tracker, and the information they re- 
ceive at each time-step is the price of the tracker corresponding to the current iteration (no agent is "cheating" and behave knowing what the "future" will be).

\subsection{Organization of the simulations}

Simulations are organized in two steps:

Stage 1: consists in the selection of the best performing agents, compared to the benchmark agent. This test consists in 10 simulations based on random subsamples from historical CAC40 observations (01/1988-07/1996). At the beginning of each simulation $(t=0)$, Family Agents create Strategic Agents. Then Strategic Agents begin to compete against the Buy \& Hold Agent. Then Family Agents rank their belonging sub-population of Strategic Agents, comparing their performances to that of the benchmark agent. Once the 10 simulations have been processed, Strategic Agents having out-performed the benchmark at least in 50\% of the simulations are selected. Performance is always appreciated in terms of risk-return : a Strategic Agents outperform the Buy \& Hold Agent if and only if she obtains a more than proportional return considering the risk she has been exposed during the simulation. "Risk" is calculated as the standard deviation of the agent's portfolio, "return" being the average rate of growth of her wealth.

Stage 2: consists in a generalization of the first stage on new data-historical CAC40 observations (08/1996-04/2005)-. Family Agents only create the Strategic Agents selected at the first step. Then the same competitive process against the Buy \& Hold Agent is processed again but on 200 sub-samples of the new data. In this last stage, Family Agents select Strategic Agents having out-performed the benchmark at least in $75 \%$ of the simulations.

We then apply a statistical procedure called Bootstrap Reality Check proposed by White (2000) to decide whether or not the "winners" in this last race have outperformed the benchmark, that is, if they have out-performed the market exploiting weak-form inefficiencies.

\section{Results}

We have selected in this short version some basic results among a large set of intersting experimental outcomes that we will describe in the full paper; a first series of figures reports part of them, directly linked to the simulations presented previously:

Results for Stage 1: 6.057 Strategic Agents have out-performed the Buy \& Hold Agent in more than $50 \%$ of the simulations. They come from all the Family Agents. The selection rate over the initial population is between $5.10^{-5 \%}$ and $1.7 \%$, which is very low, but "as expected". One has to keep in mind that our procedure involves a huge number of agents; it is perfectly normal than some of them seem to perform well at this initial step. 
Results for Stage 2: After the second selection process, only 19 Strategic Agents have out-performed the benchmark agent. They all come from two Family Agents : "Rectangle Trader" and "Variation Trader". Some of them have outperformed the benchmark agent for each of the 200 simulations.

We have then applied carefully White's Reality Check over 500 bootstrap series to control potential spurious results. Although the simulations seem to be very harsh in terms of selectivity for "good candidates", we cannot reject the null "The best Strategic Agents cannot out-perform the Buy \& Hold Agent" at ordinary p-values $p$-value $=28.2 \%$ - . Therefore we cannot reject the initial weak-form EMH and cannot report evident market inefficiencies for those data with basic Strategic Agents using simple trading rules. This result seems therefore to be a strong support for the weakform efficiency of the French Market.

Nevertheless, two interesting points have to be underlined here :

- We have applied the same experimental process using randomly generated data (CAC40 having been replaced with a computerized index following a "random walk"). By construction, these data do not "hide" any useful information at date $t$ allowing to predict $t+1$. Thus, they perfectly replicate the behavior of an efficient market index. No Strategic Agents is able in this last simulated context to pass the filters we have settled-up, while it is easily done by many of them on real-world data. This is a first troubling result.

- The last point is related to the performance of the Meta-Agents that could allow us to reject $H_{0}$, but this last point has still to be investigated further.

In the full version, we will show how our MAS can mimic complex real-world investors and achieve results that can challenge seriously the weak-form EMH.

\section{References}

Brock, W., J. LAKonishock, And B. LeBARON (1992): "Simple Technical Trading Rules and the Stochastic Properties of Stock Returns," Journal of Finance.

Detry, P., AND P. Gregoire (2001): "Other Evidences of the Predictive Power of Technical Analysis: the Moving-Average Rules on European Indices," in Proceedings of the Lugano European Financial Management Association Conference.

FAMA, E. (1970): "Efficient Capital Markets: A Review of Theory and Empirical Work," Journal of Finance.

Jensen, M., And G. Benington (1969): "Random Walks and Technical Theories: Some Additional Evidence," Journal of Finance.

MALKIEL, B. (2003): "The Efficient Market Hypothesis and Its Critics," Journal of Economic Perspectives.

PARK, C., AND S. IRWIN (2004): “The profitability of Technical Analysis," AgMAS project Research Report 2004-4.

SAmuelson, P. (1965): "Proof That Properly Anticipated Prices Fluctuate Randomly," Industrial Management Review.

SHILlER, R. J. (2003): "From Efficient Markets Theory to Behavioral Finance," Journal of Economic Perspectives.

White, H. (2000): “A Reality Check for Data Snooping," Econometrica.

Wooldridge, M. (2002): An Introduction to MultiAgent Systems. John Wiley and sons. 